ment was vast. In 1950 it was estimated that there were not more than 100 and probably fewer than 60 everglade kites in Florida. In April 1966, sixteen kites were seen in conservation area 2 . Four were known to be present on Lake Okeechobee at that time, thus indicating a minimum population of twenty birds in 1966.

Stieglitz and Thompson conclude that the development of the once vast freshwater marshes of Florida has probably made a greater contribution to the reduction of the range and numbers of the kite than any other factor. Only 1,537 square miles (56 per cent) of the 2,746 square miles of original everglades remain today in a near-natural condition. It is not inconceivable that periodic drought, either man-made or natural, may completely eliminate all suitable kite habitat. Other possible limiting factors include the effects of inbreeding in a population of fewer than 25 birds. Relatively low hatching success reported recently supports this theory.

With proper management and complete protection, the everglade kite population may increase to the point where there is no danger of extinction. In a concluding paragraph, however, the authors mention that the remaining freshwater marshes in Florida are still threatened. Recently, for example, the conservation areas 1,2 and 3, which contain some of the best remaining kite habitat, have been proposed as sites for oil pipelines, roads, TV towers, oil exploration and further flood control development. "These freshwater marshes have a high value not only for the kite but also for numerous other species, including some others considered rare or endangered. Strong efforts should be made to maintain these areas in their present condition and to prevent additional destructive inroads."

\section{Winds over Jamaica}

ONE of the most fruitful ways of measuring winds in the upper atmosphere is by using radar to track the drift of trails of ionization left behind by meteors as they burn up in the atmosphere, usually at heights between 80 and $110 \mathrm{~km}$. So far, radar measurements of meteor trail drifts have been carried out only at high latitude sites, but an experiment which will extend the measurements to low latitudes is planned by the University of Sheffield and the University of the West Indies. The joint experiment, which involves setting up a meteor radar at a site in Jamaica, is financed by a grant of $£ 8,000$ awarded to Professor T. R. Kaiser of the University of Sheffield by the Science Research Council. The radar being built in Jamaica is based on windmeasuring equipment which has been operating at Sheffield since 1964.

The essence of the method is that when particles of interplanetary dust enter the atmosphere-at speeds which may be as high as $72 \mathrm{~km} / \mathrm{s}$ - they dissipate their kinetic energy in the form of heat, light and ionization by collisions with atmospheric molecules. If the light is bright enough, the meteor is visible as a shooting star. The trail of ionization remains after the dust particle has burnt up-maybe for only a fraction of a second, but occasionally for much longer-and reflects radio waves. In fact, spurious echoes from meteor trails were often a source of confusion for radar operators during the Second World War, until their true nature was recognized. As the trail of ionization drifts with the wind in the upper atmosphere, the frequency of the returned signal is shifted by the Doppler effect, and by comparing the frequency of the returned signal with the signal transmitted by the radar, the drift velocity is measured.

Although few meteors are bright enough to be seen as shooting stars, many meteors too faint to be seen with the naked eye leave sufficient ionization to make a wind measurement possible, and echoes lasting only fractions of a second can give a value for the wind velocity. This is where the meteor method scores over photographic measurements of the drift of vapour trails released by high altitude rockets, the other important technique for upper atmosphere wind measurements. Rocket measurements can only be made at twilight, when the trail is illuminated by sunlight against a dark background, or at night using a self-luminous trail. On the other hand, interplanetary dust is entering the atmosphere all the time, so the radio meteor method can be used by day or night. The tendency has been to use vapour trail photographs to find in detail how the wind velocity depends on height in the atmosphere, at some instant of time, and radio meteor methods to follow the way the wind changes throughout the day and the seasons.

Following the pioneer meteor experiments to measure winds carried out at Jodrell Bank in the 1950s, when radio investigations of meteors were the mainstay of the research there, a number of radars to track meteor drifts have been built; at Sheffield, at Kharkov, in France, the United States and Australasia. Physicists studying wind motions in the upper atmosphere have long recognized the need to extend these measurements to other than high latitudes, and the equipment now being built in Jamaica - at $20^{\circ} \mathrm{N}$., the first low latitude station-is the first step in this direction. World-wide coverage may be further extended in a few years by the Meteorological Office, which is thinking of setting up equipment in the equatorial region to measure meteor drifts. The Meteorological Office is interested in building a radar, also based on the Sheffield pattern, which will first of all be operated in the United Kingdom, but if all goes well will be transferred to Gan, an island in the Indian Ocean close to the equator.

\section{More about Hyperoxia}

\section{from our Microbiology Correspondent}

A PRevious commentary in this column (Nature, 216, 962 ; 1967) discussed the effects of hyperbaric oxygen on the metabolism of euglenoid algae. Hyperoxia in these eucaryotic cells reflected mitochondrial lesions that disturbed electron transport. The influence of increased oxygen tensions on living cells is, however, complex and may be modified by other culture parameters such as the nutritional status of the medium. In Euglena gracilis, for example, hyperoxia is manifest only when growth was made in a low-phosphate medium. In recent years several important studies have been made of the effects of increased oxygen tensions on aerobic, pathogenic bacteria, both in vitro and in pathological conditions such as wound and burn infections. Subsequently, Gottlieb and his colleagues have turned their attention to the effects of hyperbaric oxygen on aerobic-facultative anaerobic, intestinal pathogens such as Salmonella, Shigella and Vibrio. Gottleib and Pakman (J. Bact., 95, 1003; 1968) have 